
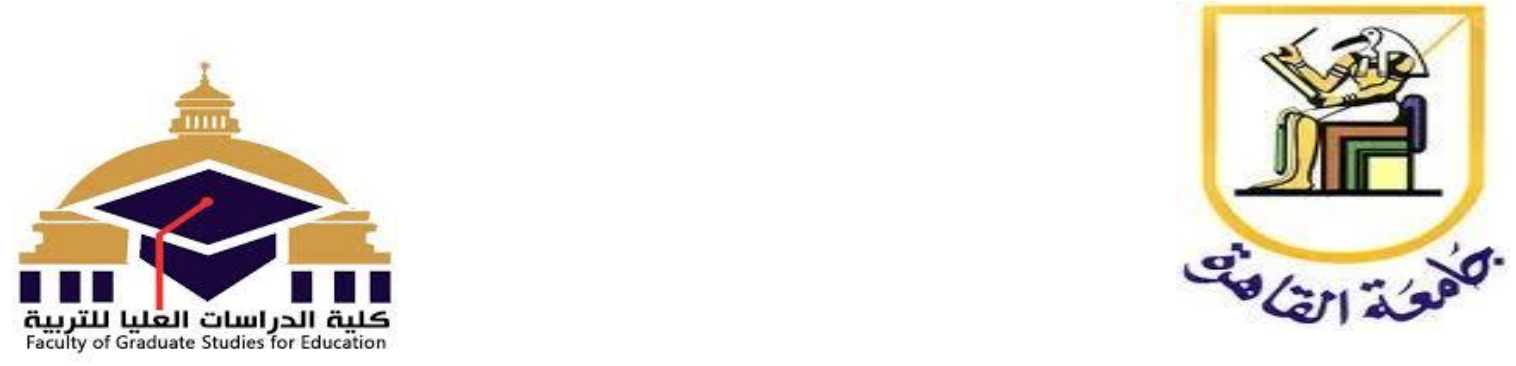

قسم التربية الخاصة

برنامج لتنمية النطق للأطفال ذوي اضطراب طيف التوحد غير اللفظين

A program for Developing the Articulation of Children with Nonverbal Autism Spectrum Disorder

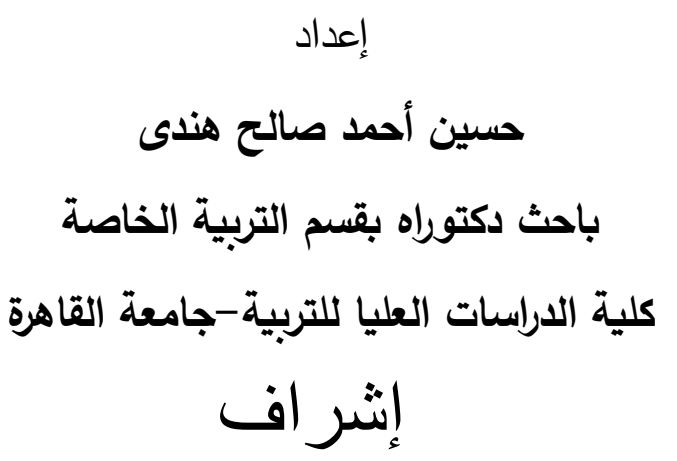

\section{الأستاذة الدكتورة}

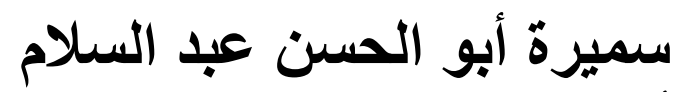

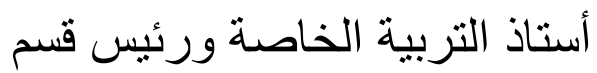

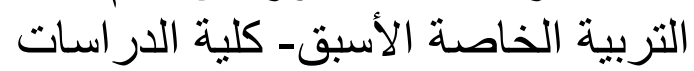

العليا للتربية - جامعة القاهرة

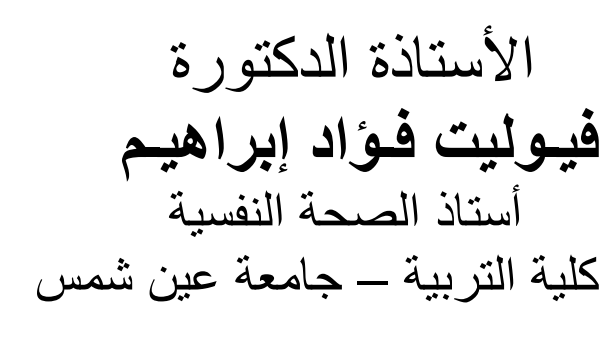

الأستاذة الدكتورة

فيوليت فوال إيراهيم

أستاذ الصحة النفسية

كلية التربية - جامعة عين شمس 


\section{برنامج لتنمية النطق للأطفال ذوى اضطراب طيف التوحد غير اللفظيين}

أ. حسين أحمد صالح هندى و أ.د سميرة أبو الحسن عبد السلام و أ.د فيوليت فؤاد إبراهيم

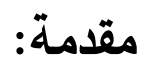

لقد شهد العالم تطوراً كبيراً فى ميدان التربية الخاصة، ولعل أبرز هذه التطورات تلك التى حصلت فى مجال فئة الأطفال ذوى اضطراب طيف التوحد Autism Spectrum Disorder سواء فيما يتعلق بقضايا الدفاهيم والتسميات، وأدوات وطرق التقييم والتشخيص الفارق، إلى جانب البرامج والطرق العلاجية الخاصة بتلك الفئة من الأطفال، واضطراب طيف التوحد ASD هو اضطراب فى النمو العصبى، يتسم بمحدودية التواصل اللفظى وغير اللفظى، وقصور فى التفاعل الاجتماعى المتبادل، ومصاحب بالسلوكيات النمطية، ومحدودية الأنثطة والاهتمامات. (Tanaka et al, 2018:1) ، وقد تم الإشارة إلى هذا المصطلح لأول مره من قبل كانر Kanner وهو طبيب نفسى أمريكى ولد فى المجر سنة (1894م) ، وهو أول من قدم وصفاً تفصيلياً لأعراض هذا الاضطراب سنة (1943م). ( عبد الرحمن سليمان، 2012 : 32-33)، كما أشارت الدراسات ذات الصلة إلى أن هناك ندرة شديدة فى الدراسات Nonverbal Autism الأبحاث الخاصة بفئة الأطفال ذوى اضطراب طيف التوحد غير اللفظيين Spectrum Disorder ، سواء على صعيد الدفاهيم والتسميات، ومجالات وأدوات التقييم، أو الأسباب التى تقف حائلاً أمام اكتساب النطق لتلك الفئة من الأطفال، بالإضافة إلى البرامج الفعالة التى يمكن استخدامها لتتمية النطق لهذه الفئة الفرعية من الأطفال، وفئة الأطفال ذوى اضطراب طيف التوحد غير اللفظيين NVASD هم فئة فرعية من من مجتمع الأطفال ذوى اضطراب طيف التوحد ASD لم تستطع تطوير النطق قبل عمر (5) سنوات.(Kasari, Brady, Lord \& Flusberg, 2013) ، وتقدر Roberts, Matsuzaki, ) بحولى (25-30\%) من مجموع الأطفال ذوى اضطراب طيف التوحد .(Blaskey, Bloy, Edgar, Kim, Ku, Kuschner... \& Embick,2019:266 مثكلة البحث: يمكن بلورة مشكلة البحث الحالي في السؤال الرئيس التالي: ما فاعلية برنامج لتتمية النطق للأطفال ذوي اضطراب طيف التوحد غير اللفظيين؟ 
وينبثق عن هذا السؤال السؤالين الفرعيين التاليين:

1- - ما الفروق بين القياسين القبلى والبعدى فى النطق لاى الأطفال ذوى اضطراب طيف التوحد غير

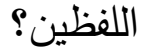

2- ما الفروق بين القياسين البعدى والتتبعى فى النطق لدى الأطفال ذوى اضطراب طيف التوحد غير اللفظيين؟

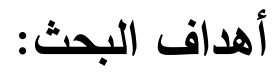
تتمثل أهداف البحث الحالي فيما يلي: 1- التحقق من فاعلية برنامج لتتمية النطق للأطفال ذوى اضطراب طيف التوحد غير اللفظين. 2- التحقق من مدى استمرارية البرنامج المستخدم فى الدراسة لدى أفراد عينة الأطفال ذوى اضطراب طيف التوحد غير اللفظيين بعد انتهاء الفترة التتبعية البالغة ثمانية أسابيع بعد انتهاء البرنامج. أهمية البحث: تتمثل أهمية البحث الحالي في جانبين أساسيين هما: - أولاً: الأهمية النظرية: 1-توفير قدر من المعلومات عن فئة الأطفال ذوى اضطراب طيف التوحد غير اللفظيين. 2-توفير قدر مـن المعلومـات النظريـة عـن النطق لفئسة الأطفـال ذوى اضـطراب طيف التوحد غير اللفظيين. - ثانياً: الأهمية التطبيقية: 1- استخدام البرنامج في تنمية النطق لاى عينات أخرى من الأطفال ذوى اضطراب طيف التوحد غير اللفظيين. 2- الاستفادة من البرنامج وفنياته كاستراتيجية للتدخل المبكر فى تتمية النطق للأطفال ذوى اضطراب طيف التوحد بصفة خاصة، وبعض الفئات الأخرى من ذوى الاحتياجات الخاصة. مصطاحات البحث: 1-النطق Articulation:هو العملية التي يتم من خلالها تشكيل الأصوات (اللبنات الأولى للكلام) الصـادر عن الجهاز الصوتي كي تظهر فى صورة رموز تتنظم فى صورة معينـة وفى أشكال وأنساق 
خاصة وفقاً لقواعد متفق عليها فى الثقافة التي ينثأ فيها القرد. (سليمان عبد الواحد، (11:2010)، وسوف يلتزم الباحث بهذا التعريف كتعريف إجرائي للنطق فى الدراسة الحالية على أساس الدرجة التى يحصل عليها المفحوص فى الاختبار المستخدم فى الدراسة الحالية. 2- اضطراب طيف التوحد غير اللفظي NASD: فئة فرعية من الأطفال ذوى اضطراب طيف التوحد Thurm, Manwaring, Swineford\& ( توقف نمو اللغة اللفظية لديهم عند بضع كلمات فقط. ASD (Farmer, 2015:97) ، ويعرف الباحث الحالى اضطراب طيف التوحد غير اللفظي NVASD إجرائياً بأنه فئة فرعية من اضطراب طيف التوحد ASD، لم تستطع اكتساب النطق، حتى عمر (8) سنوات على الرغم من تلقيهم خدمات التدخل المبكر ، ويُعرف إجرائياً وفقاً للمعايير التشخيصية لهذا الاضطراب والواردة فى الإصدار الخامس من الدليل التششخيصى للاضطرابات العقلية (DSM-5). الاطار النظري والاراسات السابقة: - اولاً: الأطفال ذوى اضطراب طيف التوحد غير اللفظيين : على الرغم مـن تتـامي مجـال البحث بالنسـبة لاضطراب طيف التوحد، فـلا تتوفر الكثير مـن المعلومات المتعلقة بالأفراد المصابين باضطراب طيف التوحد غير اللفظيين، ولم تتم دراستهم بثكل كافٍ فى غالبية الأبحاث المتعلقة بهذا الاضطراب، وفئة الأطفال ذوى اضطراب طيف التوحد غير اللفظيين هم فئة فرعية من الأطفال ذوى اضطراب طيف التوحد فقدت القدرة على التواصل اللفظى مع الآخرين وتمثل ما يقرب من( 25\% ) من مجموع الأطفال ذوى اضطراب طيف التوحد. (Wan et al, 2011:1). خصائص الأطفال ذوى اضطراب طيف التوحد غير اللفظيين: تعتبر فئة الأطفال ذوى اضطراب طيف التوحد غير اللفظيين NVASD فئة فرعية من اضطراب طيف التوحد وتحمل نفس الخصائص العامة لهذا الاضطراب، فيما عدا الخصائص اللغوية حيث أن هذه الفئة غير لآفظة، وفى السطور التالية سوف يلقى الباحث الضوء على أهم الخصائص التى تميز الأطفال ذوى اضطراب طيف التوحد عامة وفئة الأطفال ذوى اضطراب طيف التوحد غير اللفظيين بصفة خاصة.

1-الخصائص الاجتماعية والتواصلية:

يعتبر قصدور المجال الاجتماعى أحد أبرز الخصـائص السلوكية الميزة لهذا الاضطراب، كمـا يعتبر أحد أهم المعايير المستخدمة فى تثخيصه؛ إذ ترجع معظم مشكلات التفاعل الاجتماعى إلى العجز 
فى الاستجابة الاجتماعية، فقد أورد كل محمد حمدان وفيصل البلوى (2018: 106-107) أن الأطفال ذوى اضطراب طيف التوحد يتصفون بضـف واضـح فى التفاعلات الاجتماعية أثناء أنشطة اللعب المختلفة، حيث يتسم هذا اللعب بالعزلة والتى لا تشجع على التفاعل الاجتماعى البناء، كما يواجه هؤلاء الأطفال صـعوبة فى تقليـد مهارات اللعب، وربمـا يكـون لـديهم اهتمامـاً غيـر عـادى بـبعض الأثـياء واستخدامها على نحوٍ غير طبيعى، فقد يتعلقون وينشغلون بشكل مستمر بأجزاء معينـة من الأثشياء والألعاب، وقد أشـارت نتائج الدراسـات إلى أن الأطفال ذوى اضطراب طيف التوحد لديهم عجز فى مهارات التواصل الاجتماعى والتى تشتمل على مجموعة واسعة من السلوكيات اللفظية وغير اللفظية التى Wetherby, Watt, Morgan \& Shumway, ).تستخدم فى التفاعل الاجتماعى المتبادل

.$(2007: 960$

2-الخصائص اللغوية:

يعد القصـور اللغوى مـن الملامسح الثـائعة لاضطراب طيف التوحدASD، وتتفـاوت درجـات هذا القصور وأشكاله من طفل لآخر ، فقد يتمكن البعض منهم من اكتساب مهارات لغوية جيدة، ويظل البعض

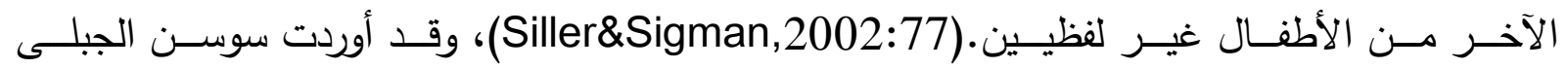
(2015:32-33 أن الفشل فى اكتساب اللغة وكافة وسائل الاتصال الأخرى قد يؤدى إلى قصور فى عمليـات الإدراك الحسى وغيرهـا مـن العمليـات العقليـة الأخـرى كالتخيـل والتـكر ومعالجـة المشكلات والاستيعاب وغيرهـا، كمـا أضـاف فلوسبيرج Flusberg)(2016:144) إلى أن هناك تباين كبير فى الخصائص اللغوية للأطفال ذوى اضطراب طيف التوحد ASD ، فمنهم من يتمتع ببنية لغوية طبيعية أو تفوق المعدل الطبيعى وذلك وفقاً لاختبارت اللغة المقننة، ومنهم من يتمكن من اكتساب الكلام بعد المرور بفترة من التأخر اللغوى ولكن يظلون لديهم محدودية فى القدرات اللغوية، ومنهم من لا يتمكن من اكتساب النطق على الرغم من تلقيهم خدمات التدخل الجيدة، وهذه الفئة يطلق عليها فئة الأطفال ذوى اضطراب طيف التوحد غير اللفظيين NVASD أو ذوى الحد اللفظى الأدنى، وأشارت نتائج العديد من الدراسات ذات الصلة إلى ضرورة الاهتمام بالتدخلات السلوكية المبكرة لما لها من تأثيرات إيجابية على اكتساب المهارت اللغوية بشقيها، وأشار نورلجين وآخرون Norellgen et al. 2015:934 إلى أن أن الأطفال ذوى اضطراب طيف التوحد ASD يمكن تقسيمهم إلى ثلاثـة فئسات وفقاً لتطور اللغـة اللفظيـة هـى ( 
اللافظة، غير اللافظة، ذوى الحد اللفظى الأدنى)، وتبلغ نسبة انتشار كل فئة على التوالى( 75 ، 15، 10 \% Chenausky, Norton, Flusberg and تقربباً، وقرر شينوسكى، نورتن، فلوسبيرج، شلاف (2018:1356) أن هناك ما يقرب من (25\%) من الأطفال ذوى اضطراب طيف التوحد غير لفظيينNonverbal، ويختلف العدد الدقيق للكلمات المنطوقة بين تلك الفئة من الأطفال، والذى

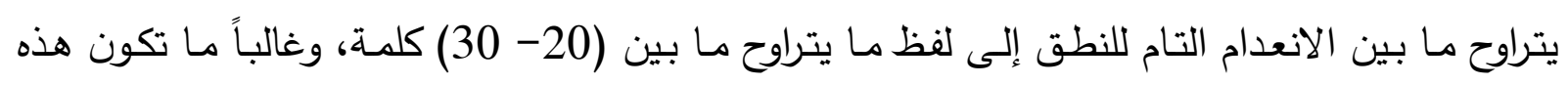
الكلمات أو العبارات مقصورة على سياقات محددة ويمكن استخدامها فقط للتواصل لتحقيق وظيفة معينة للطفل.(Kasari et al , 2013:2) ، وقد أشارت نتائج بعض الدراسات إلى ضعف احتمالية اكتساب هؤلاء الأطفال للنطق بعد بلوغ عمر (5) سنوات، بينما أشارت دراسات أخرى إلى إمكانية اكتساب النطق بعد هذا العمر • (Thurm et al, 2015)، ويعد قصور الانتباه المشترك، إلى جانب قصور

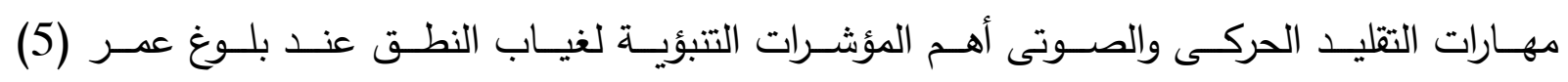
سنوات.(Thurm, Lord, Lee \& Newschaffer 2006:1721)، بينما أشار كل من ودكا، ماثى، وكالب (2013:1128) Wodka, Mathy and Kalb إلى أن كل من " شدة الأعراض الخاصـة باضطراب طيف التوحدASD، وانخفاض نسبة الذكاء خاصة نسبة الذكاء غير اللفظى، إلى جانب تدنى المشاركة الاجتماعية " تعتبر أهم العوامل التى تقف حائلاً أمام اكتساب النطق لاى تلك الفئة من الأطفال ، بينما يرى شينوسكى وآخرون (2018:1356) . Chenausky et al أن التقليد اللفظى( عدد أصوات الحروف اللفظية الككرة بشكل صحيح)، إلى جانب شدة الأعراض الخاصـة باضطراب طيف التوحد

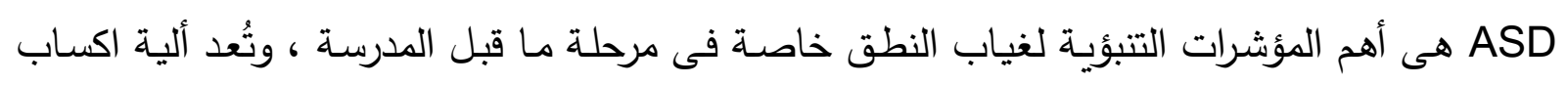
النطق للأطفال ذوى اضـراب طيف التوحد غير اللفظيين NVASD واحدة من أصعب نواحى التدخل الخاصة بتلك الفئة من الأطفال، ويلعب النطق دوراً مهماً وأساسياً فى التفاعل الاجتماعى لهؤلاء الأطفال، كما أن اكتساب النطق يعتبر أحد أهم النتائج الإيجابية التى يتوقع الحصول عليها من أجل القدرة على التى التوافق فيما بعد سواء فى مرحلة الطفولة أو المراهقة، ومن أجل ذلك يعتبر التعرف على مشكلات النطق

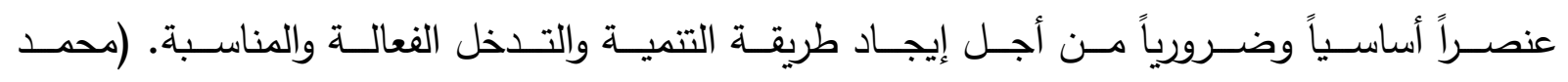
محمد، (35-30:2018). 3-الخصائص العقلية والمعرفية: 
يُظهر الأطفال ذوى اضطراب طيف التوحد تباين واسع النطاق فى القدرات العقلية. (Delehanty, (Stronach, Guthrie, Slat \& Wetherby,2018:2 وفى هذا الصدد أشار سليمان عبد الواحد (226:2009) إلى أن حوالى (30\%) من الأطفال ذوى اضطراب طيف التوحد مستوى ذكاءهم يقع فى المستوى المتوسط، وحوالى (30\%) لديهم انخفاض بسيط فى نسبة الذكاء والذى يتراوح ما بين (-70 50) درجـة، و (40\%) مـن هذه الفئة لديهم انخفاض فى نسبة الذكاء والذى يتراوح مـا بين المتوسط والثديد أى أقل مـ (50) درجة، وقرر عـادل عبدالله (2014: 145) أن هؤلاء الأطفال يعانون من مشكلات معرفية عديدة، يمكن إعتبارها خصائص معرفية لهم، ومن أمثلتها قصور فى الذاكرة والقدرات ما وراء المعرفة، ومعرفة الآخرين، ومعرفة الانفعالات، ومعرفة الذات ومهارات حل المشكلات، والتنكير المجرد، ويمكن أن تفسر أوجه القصور هذه ما يبديه هؤلاء الأفراد من مشكلات لغويـه ومشكلات التفاعل الاجتماعى، إذ أنها عادة ما تتضمن مكونات معرفية كما يحدث فى الاستخدام الاجتماعى للغة، وطرح الأسئلة والانتباه المشترك، والتقليد، واللعب الخيالى.

\section{4-الخصائص الجسمية:}

لقد أثبتت الملاحظات المباشرة للأطفال ذوى اضطراب طيف التوحد ASD أن الغالبية العظمى منهم يواجهون صعوبات فى التصرفات الحركية التى تتطلب مستويات عالية كالتخطيط، والتتسيق، والانتباه، والمحاكاه وتتفيذ حركات حسب تسلسل ما، إذ يصعب عليهم تعلم حركات متتاسقة كالرقص وفقاً لأنغام موسيقية، كما يصعب عليهم ممارسة نشاطات التوازن كالوقوف غير الطبيعى، حيث يبدو عليهم ميل جانبى، والوقوف على رؤؤس الأصابع، كما قد يكون أذرعهم ملتفة حول بعضها.(محمد حمدان، وفيصل البلوى، 2018: (128 128 ).

\section{5-(الخصائص الحسية:}

يعانى الغالبية العظمى من الأطفال ذوى اضطراب طيف التوحد ASD من قصور المعالجة الحسية، فلا يستطيعون إدراك الخبرات الحسية المختلفة التى تحدث فى البيئة، ويُحد هذا القصور من توافقهم مع المحيطين بهم ومشاركتهم الأنشطة اليومية المختلفة، وأثـارت دراسات علم النفس الفسيولوجى إلى أن هناك نمطين من قصور المعالجة الحسية لدى هؤلاء الأطفال، الأول: وهو خاص بالحساسية المفرطة تجاه المثيرات البيئية المختلفة " كتجنب اللمس، وتغطية الأذن عند سماع أصوات محددة، والحساسية 
المفرطسة للأضـواء "، أمـا الـمط الثانى: فهو نمـط ضــف الاسـتجابة الثـديد تجـاه المثيـرات البيئيـة " كمحدودية الثعور بالألم، وتجاهل الأصوات البيئية ". ( Baranek, David, Poe, Stone \&

.(Watson, 2006:591

6-السلوكيات النمطية:

تعد السلوكيات النمطية أحد المعايير الأساسية فى عملية تثخيص الأطفال ذوى اضطراب طيف التوحد ASD وذلك وفقاً للطبعة الخامسة لمعايير الدليل التشخيصى والاحصـائى للأضطرابات العقلية (DSM-5)، والتـى تتضـمن الانشـال بالحركات الإيقاعيـة التكراريـة، ومحدوديـة وصـلابة الاهتمامـات، ويُظهر مـا يقرب من (44\%) من الأطفال ذوى اضطراب طيف التوحد سلوك واحد على الأقل من السلوكيات النمطية.(Goldman, Wang, Salgado, Greene, Kim \& Rapin, 2009:30) ، ويعد هذا الجانب من الاضطراب الأكثر ألماً بالنسبة لأباء وأمهات هذه الفئة من الأطفال، وعلى الرغم من ذلك لم تحظى بالاهتمام المطلوب من قبل الباحثين والمهتمين، فالأبحاث والدراسات الخاصة بها ما زالت محدودة للغاية. (Miller, Ragozzino, Cook, Sweeney \& Mosconi, 2015:805).

\section{ثنانياً: النطق Articulation:}

يعد الكلام الذى هو أحد أدوات التواصل بين جماعات الناس، أمراً على قدرٍ كبيرٍ من الأهمية لجميع العاملين فى مجال العلاقات الإنسانية، لذلك اهتمت العلوم المختلفة بدراسته، ووضعوا لـه الرؤى المفسرة لكيفية اكتسابه، ويُعرف النطق على أنه أحد مكونات مهارة الحديث، وهو الجانب الألى الذى لا يحتاج إلىى الكثير مـن التفكير والعمليات الذهنيـة المعقدة، ويتمثل غالباً فى عبارات الترديد المكررة والقراءة الجهريـة وحفظ النصوص المكتوبـة والمسموعة وترديدها، وتعتمد هذه العملية على الكفاءة بين الذاكرة

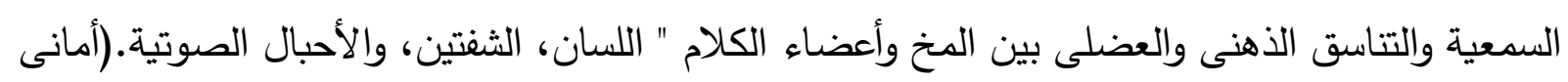
عبد الفتاح، 2018: 100)، ويعرفـه إبـراهيم الزريقـات (2005: 153) بأنـه العمليـات الحركيـة الكليـة المستخدمة فى تخطيط وإنتاج الكـلام، ويشير هذا التعريف إلى أن تعليم المهارات اللفظية هو عملية اكتسابية ناتجة عن التطور النمائى للقدرة على تحريك أعضاء النطق بطريقة دقيقة وسريعة فتعلم النطق ما هو إلا نوع محدد من التحرك الحركى. 


\section{مكونات أجهزة النطق:}

1-الجهاز التنفسى: إن وظيفة الجهاز التنفي الأساسية هى إيصال الأكسجين إلى الام والتخلص من ثانى أكسيد الكربون، فهناك علاقة بين التنفس وإنتاج الصوت ويمكن القول أنه لا تتم عملية إنتاج الصوت بثكل مناسب إذ لم تكن هناك عملية تتفس جيدة ( فالهواء يعد المادة الخام للكلام) فإذا كانت كمية الهواء الخارج من الزفير محدودة أو إذا إعترض طريق هواء الزفير أى عائق فإن إنتاج الصوت يتأثر ، كذلك إذا لم يكن هناك نوع من التآلف بين هواء الزفير وإنتاج الصوت فإن الكلمات المنتجة سوف تظهر على شكل متقطع وغير طبيعى، مما يؤدى إلى تثوه عملية النطق، ويبدأ الجهاز التفسى بفتحة الأنف، تجويف البلعوم، الحنجرة، القصبة الهوائية، والثعب الهوائية ، والحويصلات الهوائية.(سعيد

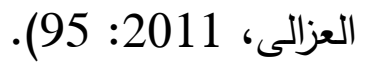

2-الجهاز الصوتى: يقع هذا الجهاز أعلى القصبة الهوائية، ويتكون من الحنجرة والأحبال الصوتية، والحنجرة عبارة عن تركيب غضروفى معقد، وهى بداية إنفصال الدذخل الهوائى عن المدخل الغذائى فى منطقة التجويف البلعومى وتحتوى الحنجرة على الغضروف الدرقى " تفاحة آدم" والغضروف الكبير والغضروف الحلقى ونجد أن الغضروف الحلقى هو أهم أجزاء الحنجرة وهو يتميز بكبره عند الرجال عنه عند النساء، وأما الغضروف الكبير فيقع فى القسم العلوى من الغضروف الحلقى وهذه الغضاريف تتحرك فى إتجاهات مختلفة وتتصل ببعضها بواسطة العضلات فيمكنها أن تعمل مشتركة مع الأجزاء الملاصقة للقصبة الهوائية أو الحنجرة، وتعتبر الأوتار الصوتية العضو الرئيسي لإصدار الصوت وهما وتران أو خيطان رفيعان من الأنسجة يمتدان يمين ويسار فتحة لسان المزمار وتتحكم غضاريف وعضلات الحنجرة فى حركتهما إلى الداخل والخارج لإحداث أصوات الكلام فعندما يتقارب الحبلين الصوتيين تحدث الاهتزازات اللازمة لإصدار الصوت، وأيضاً لمقاومة هواء الزفير الخارج من الرئتين.(سليمان عبد الواحد،45:2009).

3-جهاز الرنين: وتسمى بالتجاويف فوق المزمارية أو تجاويف ما فوق الحنجرة وتثمل تجويف الحلق ( البلعوم)، والتجويف الفهى، والتجويف الأنفى، وتلعب هذه التجاويف دوراً بارزاً ومهماً فى عملية إصدار الأصوات اللغوية وتعطى للأصوات شكلها المميز •(إيهاب الببلاوى، 2006: 89). 
4-جهاز النطق: إن الأصوات التى يصدرها جهاز الصوت تعد بمثابة المادة الخام للكلام، ويقوم جهاز النطق بتثكيلها كى تخرج فى صورة رموز صوتية متمايزة يتكون منها الكلام الثفهى المسموع.(إبراهيم

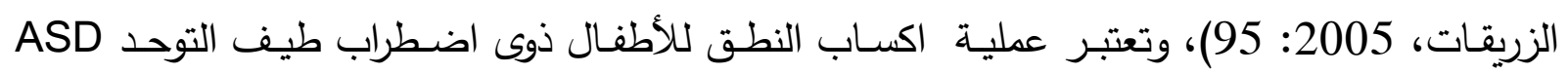

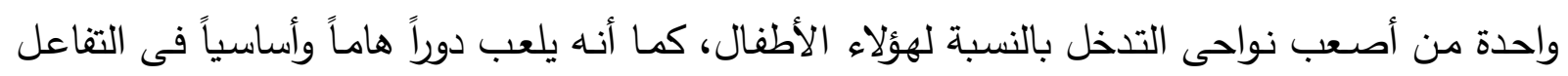

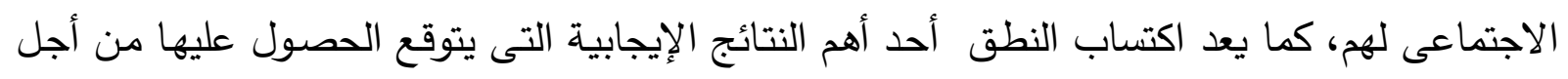

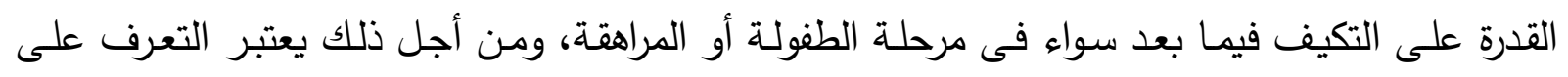

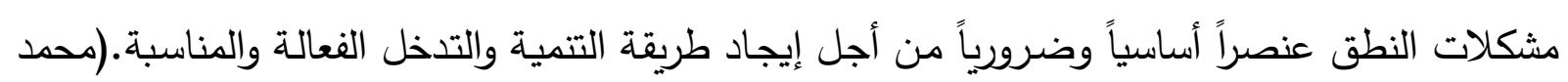

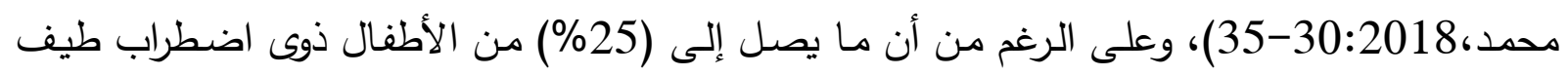

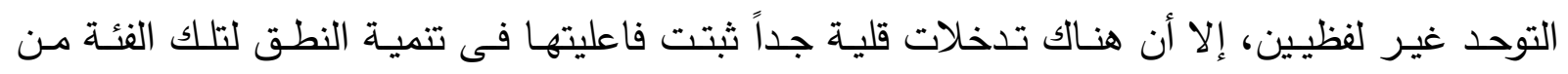
الأطفال.(Wan et all, 2011:1)، على عينة قوامها (3) أطفال ذوى اضطراب طيف التوحد غير

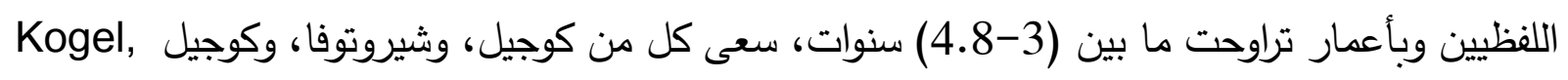
Shirotova \& Kogel (2009) النطق للأطفال عينة الدراسة، وبعد فترة تدريب امتدت نحو(8) أشهر ، بمعدل جلسة واحدة أسبوعية

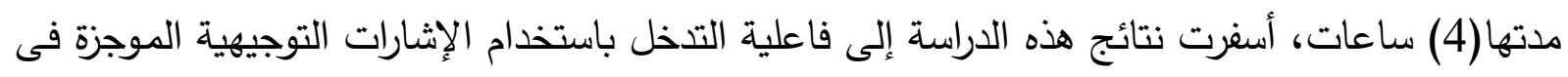
تنمية النطق للأطفال عينة الدراسة والذى تراوح ما بين (4-328) كلمة منطوقة وموظفة فى التواصل

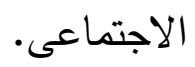

وقد أشارت العديد من الدراسات إلى أهية التدخل باستخدام الأنثطة الموسيقية الدختلفة لما لها من

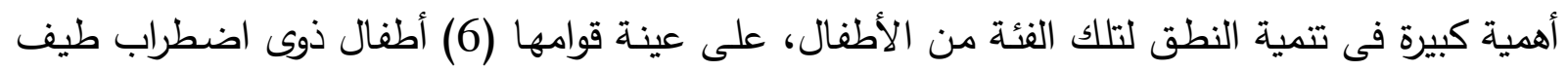

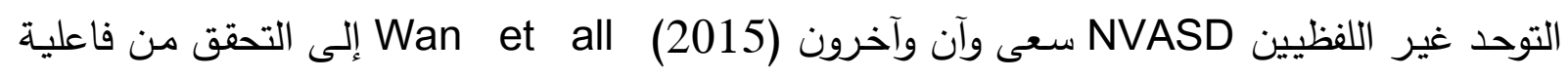
التدريب من خلال استراتيجية الخرائط الحركية السمعية على اكتساب النطق لعينة الدراسة، وبعد فترة

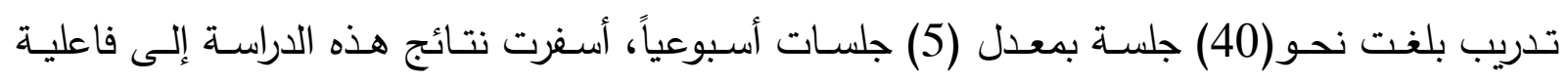

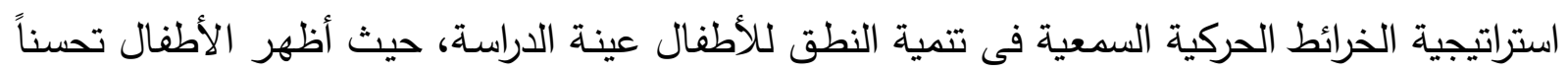
كبيراً في نطق بعض الأصوات الدفردة والكلمات، وكانت هذه الاستراتيجية بمثابة خطوة حاسمة فى تطور اللغة اللفظية للأطفال عينة الدراسة. 
Vernay, Kahina, وفى دراسة استطلاعية قام بها كل فيرناى، وكهينا، وثيرى، وجان إيف (Thierry and Jean-Yves (2017) بالتحقق من فاعلية التدخل ببعض المعينات البصرية فى تحسين النطق لعينة قوامها (4) أطفال ذوى اضطراب طيف التوحد غير اللفظيين وبأعمار تراوحت ما بين (8.7-12.1) سنة، وقد استخدم الباحثون مجموعة من المعينات البصرية مثل( الصور ، الكلمات المكتوبـة، مجموعـة من الكلمات مقسمة إلى مقاطع )، وقد عرضت هذه المعينـات من خـلال الحاسب اللوحى، وقد أسفرت نتائج هذه الدراسة إلى أن تقسيم الكلام إلى مقاطع صوتية بسيطة ذات فاعلية فى تحسين النطق للأطفال عينة الدراسة، حيث شجعت الأطفال على نطق كلمات أحادية المقطع، إلى جانب تحسن مهارة التقليد اللفظى الفورى. على عينة قوامها (2 ) من الأطفال ذوى اضطراب طيف التوحد غير اللفظيين فى مرحلة مـا قبل Drager , Postal , المدرسة، سعى كل من دراجر، وبوستال، وكارلُس، وكاستلانو، جاجليان، وجلين Carrolus , Castellano , Gagliano and Glynn(2006) فى تحسين مهارات الفهم، والتعبير اللغوى لعينة الدراسة، حيث تم إشراك الطفلين عيينة الدراسة فى أنشطة اللعب التفاعلية مع تقديم نماذج لاستخدام رموز التواصل المعززة والبديلة أثناء اللعب، وقد أسفرت نتائج هذه الدراسة عن فاعلية استراتيجية النمذجة اللغوية فى تحسن مهارات الاستيعاب والتعبير اللغوى لعينة

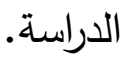

ويعد التدخل باستخدام نظم التواصل البديلة والميسرة أحد الوسائل التى يمكن استخدامها للإرتقاء بالنطق لتلك الفئة من الأطفال، وهذا ما أكدته نتائج العديد من الدراسات، على عينـة قوامها (3 ) أطفال من الأطفال ذوى اضطراب طيف التوحد غير اللفظيين وبأعمار تراوحت ما بين (3.9-7.2) سنوات، قام كل من جانز، وسيمبسون Ganz and Simpson (2004) بالتحقق من فاعلية برنامج التواصل من خـلال الصور على مهارات التواصل وتتمية النطق لعينة الدراسـة، وقد أسفرت نتائج هذه الدراسة إلى فاعلية هذا البرنامج فى زيادة الحصيلة اللغوية اللفظية للأطفال عينة الدراسة إلى جانب تعديم المهارات المكتسبة مع مجموعات متتوعة من الراشدين.

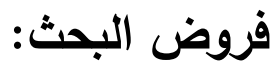
قام الباحث في ضوء مشكلة البحث و الدراسات السابقة بوضع الفرضين التالين لبحثهما: 
1- توجد فروق ذات دلالـة إحصـائية بين متوسطات رتب درجات القياسين القبلى والبعدى على مقياس النطق لاى أفراد المجموعة التجربية فى اتجاه القياس البعدى. 2- لا توجد فروق ذات دلالة إحصائية بين متوسطات رتب درجات القياسين البعدى والتتبعى على مقياس النطق لدى أفراد المجموعة التجريبية.

\section{منهج البحث وإجراء (ته:}

1

اعتمد الباحث الحالى على المنهج شبه التجريبي ذى المجموعة الواحدة والقياسات القبلي والبعدي والتتبعي، وذلك لتحقيق الهدف الرئيسي للبحث وهو تتمية النطق للأطفال ذوى اضطراب طيف التوحد غير اللفظيين من خلال البرنامج المستخدم فى البحث. 2- - عينة البحث و خصائصها:

تكونت عينـة البحث الحاليـة مـن (6) أطفـال ذوى اضـطراب طيف التوحد غير اللفظيين، مـن الحاصلين على أقل درجة على مقياس النطق، تراوحت أعمارهم الزمنية ما بين (8-12) سنة، ودرجة ذكائهم ما بين (58-67) درجة على مقياس ستانفورد بينيه الصورة الخامسة، وقد تم اختيار العينة من داخل جمعية هيا نتواصل لذوى القدرات الخاصة، بحى مدينة نصر ، بمحافظة القاهرة.

$$
\text { 3- أدوات البحث: }
$$

1-مقياس النطق للأطفال ذوى اضطراب طيف التوحد غير اللفظيين.(إعداد الباحث). 2-برنامج لتنمية النطق للأطفال ذوى اضطراب طيف التوحد غير اللفظيين.(إعداد الباحث). التحقق من الخصائص السيكومترية لمقياس النطق للأطفال ذوى اضطراب طيف التوحد غير اللفظين:

قام الباحث بالتحقق من صدق وثبات مقياس النطق للأطفال ذوى اضطراب طيف التوحد غير اللفظيين واتساقه الداخلى من خلا تقنين مقياس النطق على عينة قوامها (70) طفلاً يمثلون تلك الفئة من الأطفال، تراوحت أعمارهم ما بين (8-12) سنوات، وتم حساب معاملات الصدق والثبات للمقياس

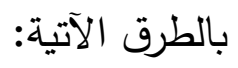
- أولاً صدق المقياس: 
للتحقق من صدق مقياس التفاعل الاجتماعى للأطفال ذوى اضطراب طيف التوحد غير اللفظين استخدم الباحث كل من الصدق التمييزي، وصدق البناء التكويني. 1-الصدق التمييزي:

وهي من أهم الطرق التي تستخدم لبيان صدق المقياس وتقوم على حساب دلالـة الفروق بين متوسطات درجات الأفراد ذوي الدرجات المرتفعة على مقياس النطق ومتوسطات درجات الأفراد ذوي الدرجات المنخفضة علي نفس المقياس وعندما تصبح لتلك الفروق دلالة احصائية واضحة فهذا يشير الي صدق المقياس كما يتضح فيما يلي:

$$
\text { جدول (1) }
$$

دلالة الفروق بين متوسطات درجات أفراد العينة على الدرجة الكلية لمقياس النطق وفقاً للنوع (ذوي الدرجات المنخفضة- ذوي الدرجات المرتفعة) $(18=ن)$

\begin{tabular}{|c|c|c|c|c|c|c|}
\hline \multirow[t]{2}{*}{ مستوى } & \multirow[t]{2}{*}{ " قيمة " " } & \multicolumn{2}{|c|}{ ذوي الارجات المرتفعة } & \multicolumn{2}{|c|}{ ذوي الارجات المنخفضة } & \\
\hline & & $\varepsilon$ & p & $\varepsilon$ & p & \\
\hline $\begin{array}{r}\text { دالة عند مستوى } \\
0,01\end{array}$ & 20.848 & 94281 & 14.7778 & 51450 & 9.5000 & الارجة الكلية \\
\hline
\end{tabular}

(*) دال غند (0.05 $(0.01 \geq \alpha)$ (*) دال عند مستوي (*)

وتوصل الباحث إلي وجود فروق دالة احصائية بين متوسطات درجات الأفراد ذوي الدرجات المنخفضة ومتوسطات درجات الأفراد ذوي الدرجات المرتفعة على الدرجة الكلية لمقياس النطق، ومن خلال الفروق التي توصل اليها الباحث يتضح من ذلك صدق المقياس. 
وفى هذه الطريقة تم حساب معامل الارتباط بين درجات الأفراد على كل عبارة والدرجة الكلية للمقياس والجدول التالى يوضح ذلك.

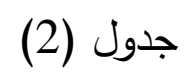

معاملات الارتباط بين العبار ات و الدرجة الكلية للمقياس النطق

$$
(70=ن)
$$

\begin{tabular}{|c|c|}
\hline معامل الارتباط & رقبحارة \\
\hline$* * 0.680$ & 1 \\
\hline$* * 0.822$ & 2 \\
\hline$* * 0.716$ & 3 \\
\hline$* * 0.691$ & 4 \\
\hline$* * 0.572$ & 5 \\
\hline$* * 0.648$ & 6 \\
\hline$* * 0.570$ & 7 \\
\hline$* * 0.456$ & 8 \\
\hline$* * 0.646$ & 9 \\
\hline
\end{tabular}

يتضح من الجدول أن جميع قيم معاملات الارتباط دالة إحصائيا عند مستوى دلالة (0.01)، وهذا يؤكد التماسك الداخلي للمقياس.

لحساب ثبات المقياس استخدام الباحث كل من طريقة ألفا - كرونباخ، وطريقة التجزئة النصفية،

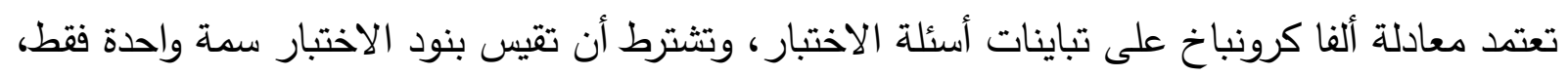
أما في طريقة التجزئة النصفية فيحاول الباحث قياس معامل الارتباط للمقياس بعد تقسيم فقراته لقسمين 
(قسمين متساويين إذا كان عدد عبارات البعد زوجي - غير متساويين إذا كان عدد عبارات البعد فردي)

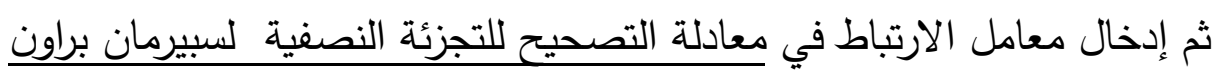

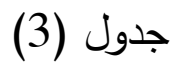

قيم معاملات الثبات بطريقة ألفا - كرونباخ وطريقة التجزئة النصفية

$$
(70=\dot{0})
$$

\begin{tabular}{|c|c|c|c|}
\hline معامل الثبات بطريقة & معامل الثبات بطريقة & عدد & \\
\hline 0.557 & 0.613 & 9 & الارجة الكلية \\
\hline
\end{tabular}

يتضح من الجدول أن قيمة معامل ألفا كرونباخ مرتفعة، وكذلك قيمة معامل الثبات بطريقة التجزئة النصفية مما يجعلنا نثق فى ثبات المقياس.

رابعاً الاساليب الإحصائية المستخدمة في البحث:

تم إجراء المعالجة الإحصائية للبيانات باستخدام الأساليب الإحصائية التالية:

1- معامل ارتباط بيرسون Pearson.

2- اختبار " ويلكوكسون " Wilcoxon Test لحساب الفروق بين متوسطات رتب درجات المجموعات

المرتبطة ، وذلك من خلال البرنامج الإحصائي للعلوم الاجتماعية SPSS.

نتائج فروض البحث ومناقشتها:

- نتائج الفرض الأول ومناقثته: لقد نص الفرض على أنه " توجد فروق ذات دلالة إحصائية بين متوسطات رتب درجات القياسين القبلى والبعدى على مقياس النطق لدى الأطفال ذوى اضطراب طيف التوحد غير اللفظيين فى اتجاه القياس البعدى ". وللتحقق من صحة هذا الفرض استخدم الباحث اختبار ويلكوكسون Wilcoxon Test اللابارامتري، والجدول التالى يوضح ذلك:

جدول (4)

متوسطات رتب درجات الأطفال ذوى اضطراب طيف التوحد غير اللفظيين 
في القياسين القبلي والبعدي على مقياس النطق

\begin{tabular}{|c|c|c|c|c|c|}
\hline مستوى الدلاكة & قيمة Z & مجموع & متوسط & العدد & نتائج القياس \\
\hline \multirow{4}{*}{ مستوى دالة عند 0.01} & \multirow{4}{*}{2.271} & 0.00 & 0.00 & 0 & الرتب السالبة \\
\hline & & 21.00 & 3.50 & 6 & الرتب الموجبة \\
\hline & & - & - & 0 & الرتب المتعادلة \\
\hline & & - & - & 6 & المجموع \\
\hline
\end{tabular}

تثير النتائج في الجدول إلى وجود فروق ذات دلالة إحصائية بين متوسطات رتب درجات الأطفال ذوى اضطراب طيف التوحد غير اللفظيين فى القياسين القبلى والبعدى فى الدرجة الكلية للمقياس، حيث كانت قيمة Z هى (2.271)، وتلك فروق دالة إحصائيا عند مستوى (0.01) فى اتجاه القياس البعدي، وهي تثير إلى نجاح وفاعلية البرنامج فى تتمية النطق للأطفال بعد تعرضهم للبرنامج، وتتقق نتائج هذا الفرض مع نتائج دراسات كل من جانز، وسيمبسون Ganz and Simpson (2004)، ودراسة كل من دراجر ، بوستال، كارلوس، كاستلانو، جاجليان، وجلين Drager , Postal , Carrolus , Castellano Vernay, ، ودراسة كل من فيرناى، كهينا، وثيرى، وجان إيف, Gagliano and Glynn(2006) Wan et all وكahina, Thierry and Jean-Yves (2017)

- نتائج الفرض الثانى ومناقشته: لقد نص الفرض على أنه " لا توجد فروق ذات دلالة إحصائية بين متوسطات رتب درجات القياسين البعدى والتتبعى لدقياس النطق لدى الأطفال ذوى اضطراب طيف التوحد غير اللفظيين.

وللتحقق من صحة هذا الفرض استخدم الباحث اختبار ويلكوكسون Wilcoxon Test اللابارامتري، والجدول التالى يوضح ذلك:

جدول (5)

متوسطات رتب درجات الأطفال ذوى اضطراب طيف التوحد غير اللفظيين في القياسين البعدي والتتبعى على مقياس النطق 


\begin{tabular}{|c|c|c|c|c|c|c|}
\hline مستوى الالالة & قيمة Z & مجموع الرتب & متوسط & العدد & نتائج القياس & \\
\hline \multirow{4}{*}{ غير دالة } & \multirow{4}{*}{0.000} & 0.00 & 0.00 & 0 & الرتب السالبة & \multirow{4}{*}{ الدرجة الكلية } \\
\hline & & 0.00 & 0.00 & 0 & الرتب الموجبة & \\
\hline & & & & 6 & الرتب المتعادلة & \\
\hline & & & & 6 & المجموع & \\
\hline
\end{tabular}

تثير النتائج في الجدول إلى عدم وجود فروق ذات دلالة إحصائية بين متوسطات رتب درجات الأطفال ذوى اضطراب طيف التوحد غير اللفظيين في القياسين البعدى والتتبعي على الدرجة الكلية لمقياس النطق، حيث كانت قيمة Z هى (0.000) وتلك فروق غير دالة إحصائيا، مما يؤكد بقاء أثر وفاعلية برنامج تتمية النطق.

\section{التوصيات التربويةة:}

انطلاقاً مما توصل إليه نتائج البحث الحالى من نتائج يوصى الباحث بما يلى: • إلقاء الضوء بمزيد من الدراسات الخاصة بفئة الأطفال ذوى اضطراب طيف التوحد غير اللفظيين، خاصة مجال القياس والتقييم لـعرفة نواحى القوة والضعف لديهم، ومن ثم بناء أطر لبرامج تدخل فعالة تعمل على الارتقاء بنواحى الضعف المختلفة خاصة مجال تتمية اللغة اللفظية.

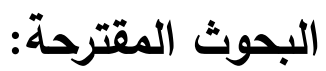

\section{استكمالاً للجهد الذى بذل فى البحث الحالى يقترح الباحث إجراء البحث التالي:}

• برنامج تدخل مبكر لتتمية النطق والمبآدأة الاجتماعية للأطفال ذوى اضطراب طيف التوحد وأثره على تفاعلهم الاجتماعى. 
أمانى عبد الفتاح على (2018). مهارات الاتصال والتفاعل والعلاقات الإنسانية. القاهرة: مكتبة الأنجلو.

إيهاب عبد العزيز البيلاوى (2006).اضطرابات التواصل. الرياض: مكتبة الزهراء.

إبـراهيم عبدالله الزريقـات (2005). اضطرابات الكـلام واللغـة " التشـخيص والعـلاجة".عمـان: دار

الفكر

سعيد كمال عبد الحميد العزالى (2011).اضطرابات النطق والكلام التثخيص والعلاج.عمان: دار

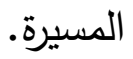

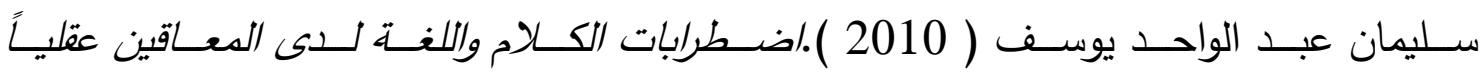
والتوحديين.القاهرة: إيتراك للطباعة والنشر .

سوسن شاكر الجبلى (2015). التوحد الطفولى ( أسبابه - خصائصسه - تشخيصسه - علاج). دمشق، سوريا: دار رسلان

عادل عبدالله محد (2014). مدخل إلى اضطراب التوحد (النظرية والتثخيص وأساليب الرعاية). القاهرة: الدار المصرية اللبنانية.

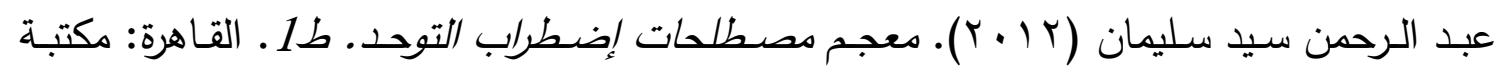
الأنجلو المصرية.

محمد أكرم حمدان؛ فيصل ناصـر البلوى (2018 ).اضطراب طيف التوحد (النشأة والتطـور-

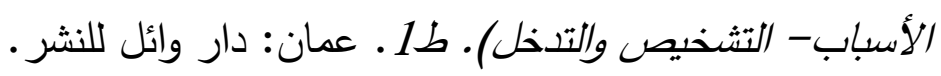

محمد رضـا السيد محمد (2018). السلوك اللفظى لدى الأطفال ذوى اضطراب طيف التوحد ( ) التهل الذاتوية ). القاهرة: مكتبة الأنجلو المصرية.

Baranek G, David F, Poe M, Stone W \& Watson L. (2006). Sensory Experiences Questionnaire:discriminating sensory features in young children with autism, developmental delays, and typical development. Journal of Child Psychology and Psychiatry, 47(6), 591-601. . Doi: 10.1111/j.14697610.2005.01546.x. 
Cadette J, Wilson C, Brady M, Dukes C \& Bennett K.(2016). The Effectiveness of Direct Instruction in Teaching Students with Autism Spectrum Disorders to Answer "Wh-" Questions. Journal of Autism and Development Disorders, 46 (9), 2968-2978. Doi: 10.1007/s10803-016-2825-2.

Chenausky K, Norton A, Flusberg H \& Schlaug G.( 2018). Behavioral Predictors of Improved Speech Output in Minimally Verbal Children with Autism. Autism Research, 11(10), 1356-1365. Retrieved from https://www.ncbi.nlm.nih.gov/pmc/articles/PMC4518704/

Delehanty A, Stronach S, Guthrie W, Slate E \& Wetherby A. (2018). Verbal and nonverbal outcomes of toddlers with and without autism spectrum disorder, language delay, and global developmental delay. Autism \& Developmental Language Impairments, 3, 1-19. Doi: 10.1177/2396941518764764.

Drager K, Postal V, Carrolus L, Castellano M, Gagliano C \& Glynn J. (2006) The Effect of Aided Language Modeling on Symbol Comprehension and Production in 2 Preschoolers with Autism. American Journal of SpeechLanguage Pathology, 15 (2), 112-125. Retrieved from https://www.pubs.asha.org/doi/abs/10.1044/1058-0360\%282006/012\%29.

Faja S \& Darling L. (2019). Variation in Restricted and Repetitive Behaviors and Interests Relates to Inhibitory Control and Shifting in Children With Autism Spectrum Disorder. Autism, 23(5),1262-1272. Doi: $10.1177 / 1362361318804192$.

Flusberg H. (2016). Risk Factors Associated With Language in Autism Spectrum Disorder: Clues to Underlying Mechanisms. Journal of Speech, Language, and Hearing Research, 59(1), 143-154. Doi: 10.1044/2015_JSLHR-L-150146.

Ganz J \& Simpson R. (2004). Effects on Communicative Requesting and Speech Development of the Picture Exchange Communication System in Children with Characteristics of Autism. Journal of Autism and Developmental Disorders, 34(4), 395-409. . Retrieved from https://www.researchgate.net/publication/8264843 Effects on Communicative Requesting and Speech Development of the Picture Exchange Communication System in Children wi th Characteristics of Autism.

Goldman, Wang, Salgado, Greene, Kim \& Rapin. (2009). Motor stereotypies in children with autism and other developmental disorders. DEVELOPMENTAL MEDICINE \& CHILD NEUROLOGY, 51(1), 30-38. Doi: 10.1111/j.1469-8749.2008.03178.x.

Kasari C, Brady N, Lord C, \& Flusberg H. (2013). Assessing the Minimally Verbal School-Aged Child with Autism Spectrum Disorder. Autism Research, 6 (6), 479-493. Doi: 10.1002/aur.1334. 
Koegel R, O'Dell M \& Dunlap G. (1988) .Producing Speech Use in Nonverbal Autistic Children by Reinforcing Attempts. Journal of Autism and Developmental Disorders, 18 (4), 525-538. . Retrieved from https://pubmed.ncbi.nlm.nih.gov/3215880/

Koegel R, Vernon W \& Koegel L. (2009). Improving Social Initiations in Young Children with Autism Using Reinforcers with Embedded Social Interactions .Journal of Autism and Developmental Disorders, 39 (9), 1240-1251. Retrieved from https://www.ncbi.nlm.nih.gov/pmc/articles/PMC2759868/.

Leekam S \& Prior M. (2011). Restricted and Repetitive Behaviors in Autism Spectrum Disorders: A Review of Research in the Last Decade. Psychological Bulletin, 13(7), 562-593. Doi: 10.1037/a0023341.

Lowe J, Werling D, Constantino J, Cantor R \& Geschwind D. (2015). Social Responsiveness, an Autism Endophenotype: Genomewide Significant Linkage to Two Regions on Chromosome 8. The American Journal of Psychiatry, 172(3), 266-275. Doi: 10.1176/appi.ajp.2014.14050576.

Miller H, Ragozzino M, Cook E, Sweeney J \& Mosconi M. (2015). Cognitive Set Shifting Deficits and Their Relationship to Repetitive Behaviors in Autism Spectrum Disorder. Journal of Autism and Developmental Disorders , 45, 805-815. . Doi: 10.1007/s10803-014-2244-1.

Norrelgen F, Fernell E, Eriksson M, Hedvall A, Persson C, Sjölin M, Gillberg C \& Kjellmer L.(2015). Children with Autism Spectrum Disorders Who Do Not Develop Phrase Speech in the Preschool Years. Journal of Autism, 19(8), 934-943. Doi: 10.1177/1362361314556782

Roberts L, Matsuzaki J, Blaskey L, Bloy L, Edgar C, Kim M, Ku M, Kuschner E \& Embick D.(2019). Delayed M50/M100 Evoked Response Component Latency in Minimally Verbal/Nonverbal Children Who Have Autism Spectrum Disorder. Molecular Autism, 10(34), 1-10. Doi: 10.1186/s13229019-0283-3.

Siller M \& Sigman M. (2002). The behaviors of Parents of Children with Autism Predict the Subsequent Development of Their Children's Communication. Journal of Autism and Developmental Disorders, 32(2), 77-89. Doi: 10.1023/a:1014884404276. Doi: 10.1023/a:1014884404276.

Thurm A, Lord C, Lee L \& Newschaffer C. (2006). Predictors of Language Acquisition in Preschool Children with Autism Spectrum Disorders. Journal of Autism and Developmental Disorder, 37(9), 1721-1734. Doi: 10.1007/s10803-006-0300-1. 
Thurm A, Manwaring S, Swineford L \& Farmer C.(2015). Longitudinal Study of Symptom Severity and Language in Minimally Verbal Children with Autism. Journal of Child Psychology and Psychiatry, 56(1), 97-104. Rerieved from https://www.pubmed.ncbi.nlm.nih.gov/24961159/

Vernay F, Kahina H, Thierry M \& Jean-Yves R. (2017).Self-paced segmentation of written words on a touch screen tablet promotes the oral production of nonverbal and minimally verbal children with autism". Journal of Research in Special Educational Needs, 17 (4), 265-273. Retrieved from https://www.semanticscholar.org/paper/Self-Paced-Segmentation-of-Written-Words-on-a-theVernay-Kahina/d330361d3b7d2867527ebc4bc98451ec2a318d4b.

Wan, C, Bazen L, Baars R, Libenson A, Zipse L, Zuk J, Norton A \& Schlaug G. (2011). Auditory-Motor Mapping Training as an Intervention to Facilitate Speech Output in Non-Verbal Children with Autism: A proof of Concept Study. PLoS One, 6(9), e2550. Retrieved from https://pubmed.ncbi.nlm.nih.gov/21980480/.

Wetherby M, Watt N, Morgan L \& Shumway S. (2007). Social Communication Profiles of Children with Autism Spectrum Disorders Late in the Second year of Life. Journal of Autism and Developmental Disorders, 37(5), 960975. Doi: 10.1007/s10803-006-0237-4.

Wodka E, Mathy P \& Kalb L. (2013). Predictors of Phrase and Fluent Speech in Children with Autism and Severe Language Delay. Pediatrics, 131(4), 1128-1134. Doi: 10.1542/peds.2012-2221. 